\title{
Localized edge modes in discrete photonic and phononic systems
}

\author{
(Review Article)
}

\author{
Yuri Kivshar \\ Nonlinear Physics Center, Australian National University, Canberra ACT 2601, Australia \\ ITMO University, St. Petersburg 197101, Russia \\ E-mail: ysk@internode.on.net
}

Received March 14, 2019, published online July 26, 2019

\begin{abstract}
In this Review we discuss three examples of different physical systems where the energy localization occurs at the edges due to nonlinearity, topology, or curvature. In many of such systems, the existence of localized edge modes and their properties can be verified experimentally, as for the case of arrays of weakly coupled optical waveguides.
\end{abstract}

Keywords: edge modes, nonlinearity, topology, discrete systems.

\section{Contents}

1. Introduction... 1201

2. Nonlinear edge modes .1201

3. Topological edge states

.1203

4. Modes localized due to curvature

5. Concluding remarks .1206

References. .1207

\section{Introduction}

Many research topics run at the Institute for Low Temperature Physics and Engineering during the Directorship of Prof. Boris Verkin have been devoted to theoretical and experimental studies of spatially localized modes in different types of low-dimensional solid-state and optical systems. In linear systems, localized modes are usually associated with defects such as surfaces, interfaces, or impurities, whereas in nonlinear systems localized modes appear as self-trapped states or discrete solitons (sometimes called intrinsic localized modes).

Currently, we observe a growing interest in the study of spatially localized modes in photonic systems. Indeed, as the size of active photonic devices decreases, the role of defects and surfaces in photonic structures becomes increasingly important. In optics, surface electromagnetic waves are known for many years as the waves localized at the interface separating either two homogeneous or homogeneous and periodic dielectric media [1], while nonlinear dielectric media can support different types of nonlinear guided waves localized at or near the surfaces [2]. Nonlin- ear guided waves in planar waveguiding structures have been studied extensively for last 20 years.

The paper is organized as follows. In Sec. 2, we discuss optical modes which are localized at the edge of an array of optical waveguides due to nonlinearity. Such modes exist when their power exceeds some threshold value, and they can be characterized by an effective energy describing the interaction of light with the edge waveguide. Section 3 is devoted to the study of edge modes in zigzag arrays of nanoparticles demonstrating topological properties. In such topological structures, the localization occurs due to nontrivial topological properties when the zigzag angle exceeds some critical value. Finally, in Sec. 4 we consider an example of a phononic system, such as a capped carbon nanotube, and demonstrate that curved surface of the cap can result in a strong localization of vibrational phonons. Finally, Sec. 5 concludes the paper.

\section{Nonlinear edge modes}

Surface modes are a special type of waves localized at an interface between two media. Recently, it was predicted 
theoretically and demonstrated experimentally that nonlinear self-trapping of light near the edge of a waveguide array with self-focusing nonlinearity can lead to the formation of discrete surface solitons (see, e.g., the review paper [3]). It was found that the self-trapped surface modes acquire some novel properties different from those of the discrete solitons in infinite lattices: discrete surface states can only exist above a certain threshold power and, for the same value of the power, up to two different surface modes can exist simultaneously. This can be understood as discrete optical solitons localized near the surface but experiencing a repulsive force from the surface [4].

To demonstrate the property of nonlinear localization, we study a semi-infinite array of identical, weakly coupled nonlinear optical waveguides described by the system of coupled-mode equations for the normalized mode amplitudes $E_{n}$,

$$
\begin{gathered}
i \frac{d E_{1}}{d z}+\alpha E_{1}+E_{2}+\gamma\left|E_{1}\right|^{2} E_{1}=0, \\
i \frac{d E_{n}}{d z}+\alpha E_{n}+\left(E_{n+1}+E_{n-1}\right)+\gamma\left|E_{n}\right|^{2} E_{n}=0,
\end{gathered}
$$

where in the second equation $n \geqslant 2$, propagation coordinate $z$ is normalized to intersite coupling $V$, the field amplitudes $\mathcal{E}_{n}$ are defined in terms of the actual electric fields $E_{n}$ as $\mathcal{E}_{n}=\left(2 V \lambda_{0} \eta_{0} / \pi n_{0} n_{2}\right)^{1 / 2} E_{n}$, where $\lambda_{0}$ is the freespace wavelength, $\eta_{0}$ is the free-space impedance, $\alpha$ is the normalized linear propagation constant of each waveguide, $n_{2}$ and $n_{0}$ are nonlinear and linear refractive indices of each waveguide, and $\gamma= \pm 1$ defines the type of nonlinearity: focusing or defocusing [4].

We look for stationary modes of the waveguide array in the form $E_{n}(z)=\exp (i \beta z) E_{n}$, where $\beta$ is the nonlinearityinduced shift of the propagation constant. Figures 1(a), (b) show two examples of the nonlinear localized states centered at different sites near the surface for both focusing and defocusing nonlinearities. The surface state centered at $n=1$ and shown in Fig. 1(a) (left) was predicted earlier [5]. The existence of multiple localized states near the surface and their properties have been analyzed by Molina et al. [4] who revealed an important interplay between nonlinearity and discreteness in the array and studied the effect of the lattice truncation on the stability of the surface states. In both the cases, the states in Figs. 1(a) and (b) describe a crossover regime between the modes with the maximum amplitude at the surface and the modes which are weakly affected by the presence of the surface.

Figure 1(c) shows power $P$ of the localized surface states versus the propagation constant $\beta$ for the modes in the focusing waveguides shown in Fig. 1(a), and the corresponding curves for the modes of the defocusing waveguides are mirror images. Direct numerical simulations and stability analyses confirm the validity of the VakhitovKolokolov stability criterion [6]; the instability region (that corresponds to a negative slope of the power curve) decreases as the center of the localized mode gets shifted away from the array edge.
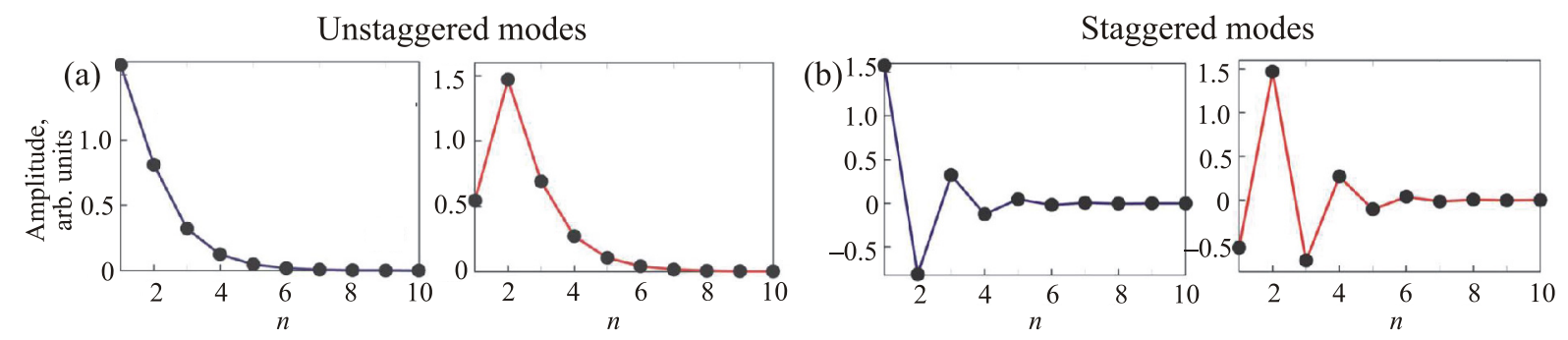

(c)
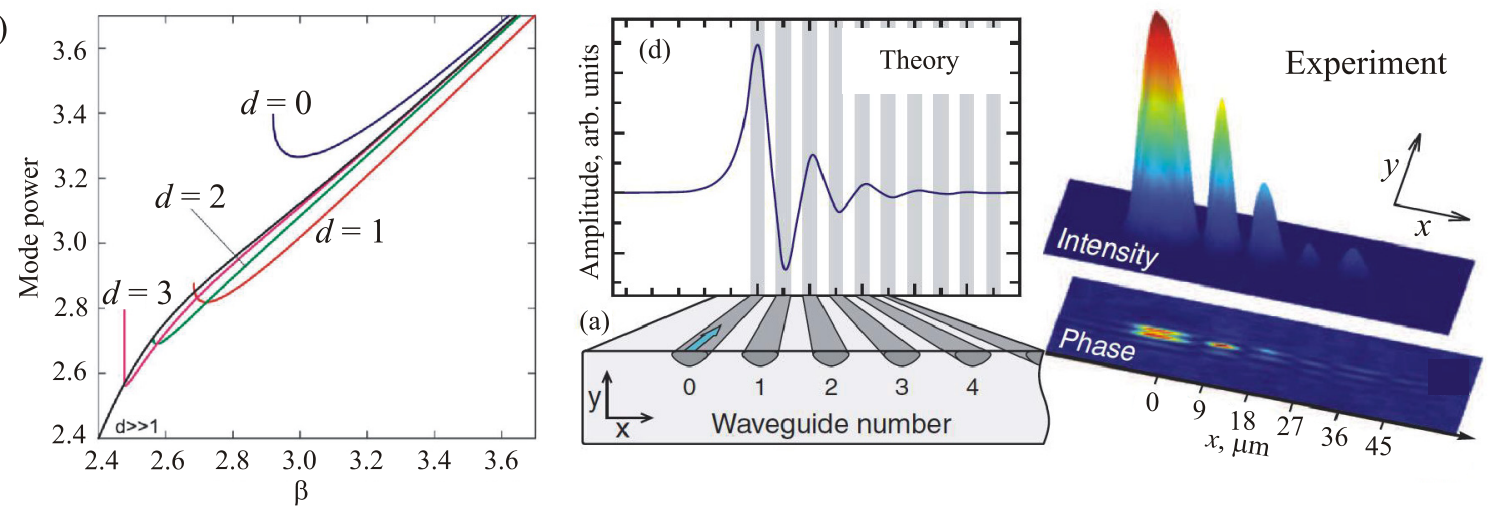

Fig. 1. (Color online) Nonlinear localized modes in discrete lattices. (a), (b) Examples of discrete surface solitons in an array of (a) focusing $(\gamma=+1$ and $\beta=+3)$ and defocusing $(\gamma=-1$ and at $\beta=-3)$ waveguides centered at the distances $d=0$ and $d=1$, respectively [4]. (c) Normalized power vs. propagation constant $\beta$ for the edge modes (discrete surface solitons) shown in (a) at the distances $d=0,1,2,3$ from the edge. (d) Theoretical prediction and experimental observation of the nonlinear edge states in an array of defocusing optical waveguides [8]. 
Shortly after the prediction of discrete optical solitons near the boundary of a self-focusing cubic nonlinear waveguide array, the first observation of these surface solitons was carried out [7]. While these surface optical solitons can be understood in terms of self-rapping of light at large enough powers, the subsequent observations of nonlinear localized states has been done for surface gap solitons at the edge of a self-defocusing $\mathrm{LiNbO}_{3}$ waveguide array [8]. In this experiment, the array consisted of 100 single-mode optical waveguides fabricated by titanium in-diffusion process in a monocrystal $x$-cut lithium niobate wafer. The $\mathrm{LiNbO}_{3}$ exhibits a strong photovoltaic effect which leads to defocusing saturable nonlinearity at visible wavelengths. At low laser power $(0.1 \mathrm{~W})$, two major effects were observed. First, due to coupling between neighboring waveguides the probe beam experiences discrete diffraction and spreads out in the horizontal plane upon propagation. Second, the beam shifts dramatically to the right indicating a strong repulsive effect of the surface. Increasing the laser power leads to spatial beam self-action through the defocusing photovoltaic nonlinearity. This nonlinearity-induced suppression of the surface repulsion leads to partial selftrapping at the surface, with a tail of intensity lobes extending into the periodic structure. A series of zero intensity points between these lobes indicates the self-induced formation of a staggered phase, as shown in Fig. 1(d).

In general, this scenario of the formation of nonlinear localized modes is rather universal. In a majority of discrete physical systems, a truncation of the system does not lead to any surface localized modes the edge or surface introduced by the truncation corresponds to a repulsive effective potential. Nonlinearity supports self-trapping and may overcome the surface repulsion, thus supporting nonlinear surfaces state with the energy exceeding a threshold.

\section{Topological edge states}

Topological insulators represent a novel class of materials with a topologically protected phase order [9]. Such materials attracted a lot of attention in the recent years due to the existence of novel types of conducting surface states in otherwise insulating bulk materials being protected by time-reversal symmetry, and they demonstrate novel phenomena such as the quantum Hall effect. Recently, the concept of topological insulators became attractive in optics, and different types of electromagnetic topological states have been realized experimentally [10]. The first electromagnetic topological edge states were demonstrated for microwaves, and later such novel photonic states were also realized at optical frequencies in coupled optical waveguides and optical lattices (see the review paper [10] and references therein).

Recently, it was predicted theoretically that a novel type of topological edge states can be realized in the subwavelength regime for a zigzag array of plasmonic nanoparticles [11]. Nontrivial topological properties of these plasmonic edge states have been studied in the framework of the coupleddipole approximation and also by direct numerical simulations of Maxwell's equations. Later, the topological properties of zigzag arrays of dielectric particles have been studied experimentally for microwaves [12] and optics [13]. Very recently, unusual nonlinear properties due to topological phases in such arrays have been revealed experimentally in the third-harmonic generation [14].

To describe the topological properties of zigzag arrays, we consider a general model originating from two major assumptions [12]: (i) polarization-dependent interaction between resonant modes of its structural elements, and (ii) a zigzag shape of the array [Fig. 2(a)]. The structure Hamiltonian can be presented in a rather general form [12]

$$
H=\sum_{j, v} E_{0} a_{j v}^{\dagger} a_{j v}+\sum_{\left\langle j, j^{\prime}\right\rangle, v, v^{\prime}} a_{j v}^{\dagger} V_{v v^{\prime}}^{\left(j, j^{\prime}\right)} a_{j^{\prime} v^{\prime}},
$$

where $E_{0}$ is the resonance energy, the indices $j$ and $j^{\prime}$ label the particles, and $\left\langle j, j^{\prime}\right\rangle$ are the nearest neighbors. We are interested only in the states excited at the normal light incidence upon the zigzag plane $(x y)$, i.e., the mode polarization $v$ can be $x$ or $y$ for dipole resonances and $v=x z, y z$ for the quadrupole resonances.

In this Hamiltonian, $V_{v v}^{\left(j, j^{\prime}\right)}=t_{\|} \cos ^{2} \psi+t_{\perp} \sin ^{2} \psi$ (with $v=x$ or $x z$ ), $V_{v v}^{\left(j, j^{\prime}\right)}=t_{\|} \sin ^{2} \psi+t_{\perp} \cos ^{2} \psi$ (with $v=y$ or $y z$ ) and $V_{v v^{\prime}}^{\left(j, j^{\prime}\right)}=\left(t_{\|}-t_{\perp}\right) \sin \psi \cos \psi$ for $v \neq v^{\prime}$, where $\psi$ is the azimuth angle of the vector $\mathbf{R}=\mathbf{r}_{j}-\mathbf{r}_{j^{\prime}}$. Here, $t_{\|}$and $t_{\perp}$ are the coupling constants of the modes co- and crosspolarized with respect to the link $\mathbf{R}$; for quadrupolequadrupole interaction $t_{\|} / t_{\perp}=-4$ and for dipole-dipole interaction $t_{\|} / t_{\perp}=-2$.

The calculated spectrum of the Hamiltonian (3) for the finite chain with $N=60$ particles is shown in Fig. 2(c) as function of the angle $\theta$ between two consecutive links in the zigzag. The value of $\theta$ equal to $\pi$ corresponds to straight line when the two polarizations $v$ are decoupled, the system is not gapped and no edge states are present. The case $\theta \ll 1$ corresponds to degenerate zigzag with very acute angles. For $|\theta-\pi / 2|<\arcsin |2 t / \Delta|$ a spectral gap appears with a pair of eigenstates, one localized at the left edge and one at the right edge. All further details can be found in an earlier paper [12].

Topological edge states in our system rely strongly on the polarization degeneracy of the eigenmodes of individual particles, and thus the problem is more general and much richer than the celebrated scalar Su-SchriefferHeeger (SSH) model for polyacetylene [15]. Although optical analogues of the SSH model were extensively studied in dimer superlattices, its topological properties have been fully understood only recently [16]. In our system, contrary to the SSH model, all the particles are the same and a pair of zero-energy states is present for both odd and even number of particles $N$. Localization degree increases 
(a)
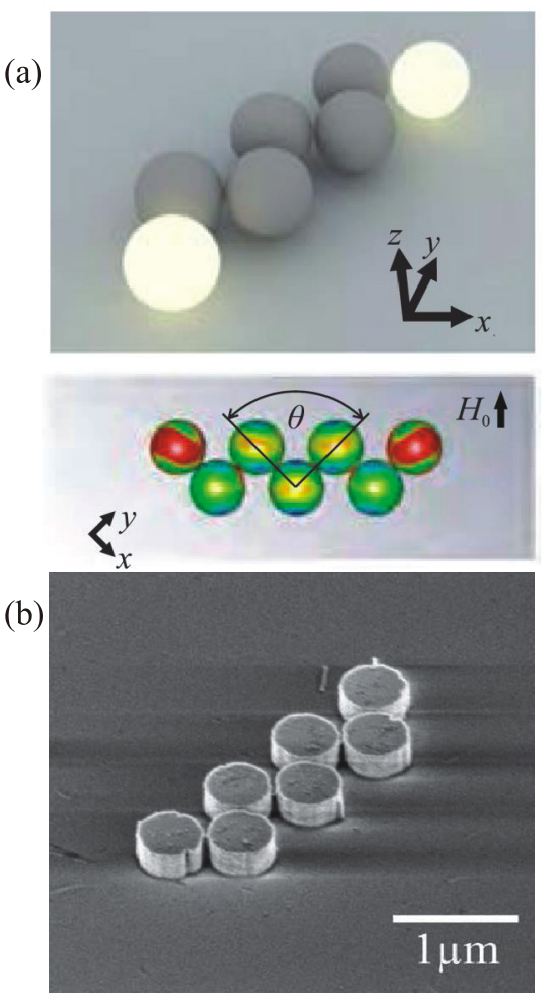

(c)

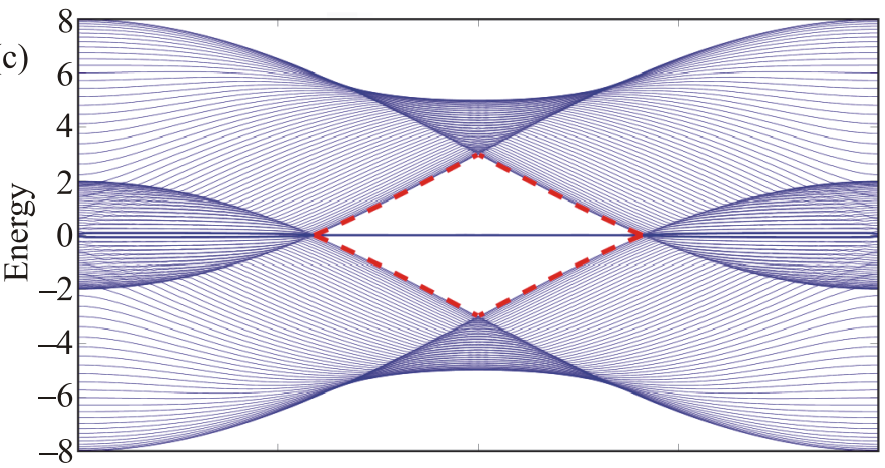

Bond angle, $\theta$

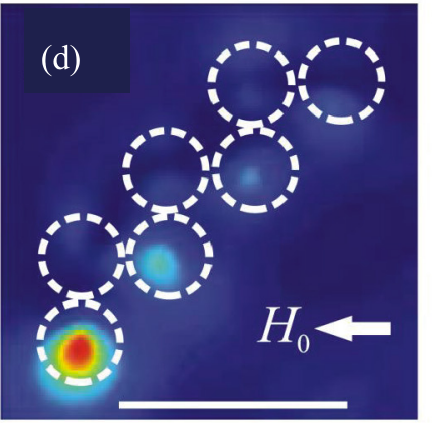

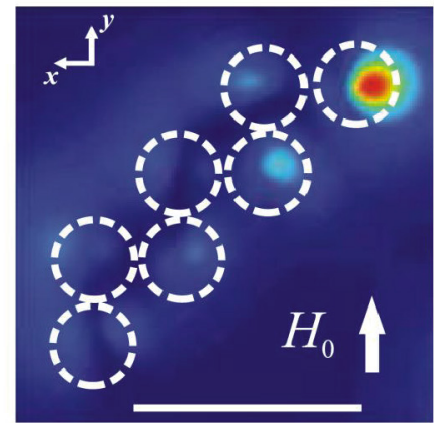

Fig. 2. (Color online) Topological edge modes in arrays of Mie-resonant dielectric particles. (a) Artist's view of a zigzag array of topologically nontrivial zigzag array of dielectric spheres. (b) A zigzag array of silicon nanodisks fabricated experimentally [13]. (c) Energy spectrum of the zigzag array calculated as the function of the bond angle $\theta$. Dashed red lines mark the region where the topological edge states can exist. (d) Experimental observation of topological edge states at the frequency of the magnetic dipole resonance [12] excited by the different orientations of the magnetic component of the external wave.

with the number of particles. For odd $N$ and $\theta=\pi / 2$ the edge states are co-polarized, and for even $N$ they are cross-polarized. This opens a possibility to selectively excite left or right edge of the same structure by varying the incident wave polarization.

Being inspired by this numerical demonstration of the edge excitation in the zigzag array, Slobozhanyuk et al. performed the proof-of-concept experiments in the microwave frequency range. To mimic the electromagnetic properties of silicon nanoparticles, they employed $\mathrm{MgO}-\mathrm{TiO}_{2}$ ceramic spheres that are characterized by a dielectric constant of 15 and small dielectric loss factor in the $4-10 \mathrm{GHz}$ frequency range [17]. The sphere radius is equal to $R=7.5 \mathrm{~mm}$ and the spheres are touching each other. The experimental results are summarized in Fig. 2(d). For chosen parameters the magnetic quadrupole resonance frequency is equal to $f=7.2 \mathrm{GHz}$.

Next, Slobozhanyuk et al. [12] proceeded to the analysis of the near-field maps measured in the close vicinity of the dielectric zigzag array at the magnetic quadrupole resonance. In order to approximate the plane wave excitation, they utilized a rectangular horn antenna. It is connected to the transmitting port of a vector network analyzer (Agilent E8362C). They also used an automatic mechanical near-field scanning device and an electric field probe connected to the receiving port of the analyzer. The probe is oriented normally with respect to the interface of the structure, and we measure $E_{z}$ component of the electric field. The near field was scanned at the $1 \mathrm{~mm}$ distance from the back interface of the zigzag array to avoid the contact between the probe and the sample. The polarization dependence of the structure response is examined by rotating the source antenna.

Two images in Fig. 2(d) show the electric field maps for different angles $\phi$ between the polarization direction of the incident magnetic field $\mathbf{H}_{0}$ and the $x$ axis. The maps present a direct confirmation of the edge excitation in the structure at the magnetic quadrupole resonance. The relative intensity of the field above the edges can be switched by rotating the incident wave polarization. For $\phi=0$ or $90^{\circ}$ polarizations, only the first or last sphere are excited, respectively. These results directly demonstrate the difference between our polarization sensitive system and the scalar SSH model. Based on the simplified analysis [11], the edge mode excitation scenario is different for even number of particles, where both edges are excited identically with polarization-dependent intensities. Similar behavior is observed experimentally for six dielectric particles at the magnetic quadrupole resonance. The experimental results of Fig. 2(d) are in full qualitative agreement with the predictions of the symmetry analysis of the Hamiltonian (3). 
Thus, Slobozhanyuk et al. [12] proposed and experimentally demonstrated a novel class of photonic topological edge states realized in the zigzag arrays of subwavelength dielectric resonant structures. They have demonstrated experimentally selective excitation of topological edge states by adjusting the polarization of the incident wave, and we have clarified the difference between topologically trivial and nontrivial electromagnetic modes in such arrays. We believe that the study of topological polarization-entangled eigenmodes suggests a new way for engineering the properties of subwavelength structures and all-dielectric metamaterials for novel applications in nanophotonics.

Very recently, Kruk et al. [14] employed this system for the observation of a third-harmonic signal from a topologically nontrivial zigzag array of dielectric nanoparticles and the demonstration of strong enhancement of the nonlinear photon generation at the edge states of the array. The signal enhancement is due to the interaction between the Mie resonances of silicon nanoparticles and the topological localization of the electric field at the edges. The system is also robust against various perturbations and structural defects.

The existence of a topological phase in a zigzag array when the zigzag angle is selected in the spectral gap, allows the edge modes appear even in the case of truncated arrays of identical particles. This is somewhat controversial property is a result of the exotic topology of the array that can be characterized by a nonzero winding number [12], in a contrast with trivial arrays.

\section{Modes localized due to curvature}

It has been well established that surfaces can often support a special class of spatially localized surface states [18], which have been studied in many branches of physics, including electrons in crystals, surface phonons, surface polaritons, and optical surface modes in waveguide arrays. However, a detailed analysis and direct observation of highly localized excitations at the atomic level remains rather difficult. A broad class of such atomic systems is presented by carbon nanotubes [19], which support different types of phononic localized modes.

Here, we discuss phononic surface states and consider two types of single-walled carbon nanotubes, which are known to have the smallest diameters. The structure of the nanotubes is shown in Fig. 3. A zigzag $(m, 0)$ single-wall carbon nanotubes with the indices $m=6$ has $L>2$ transverse segments consisting of $N=m+2 m L+m$ carbon atoms (each tip has $m$ atoms, and each segment has $2 m$ atoms). The structures with the smallest number of atoms $N=36$ for $m=6$ at $L=2$ transform into fullerene $\mathrm{C}_{36}$.

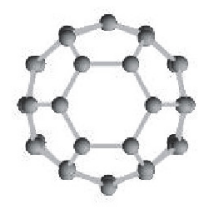

$(6,0)$

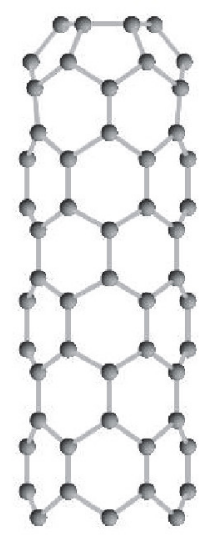

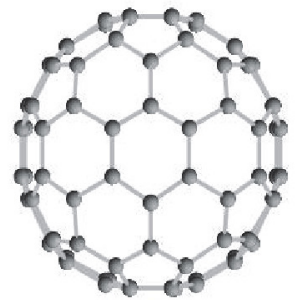

$(6,6)$

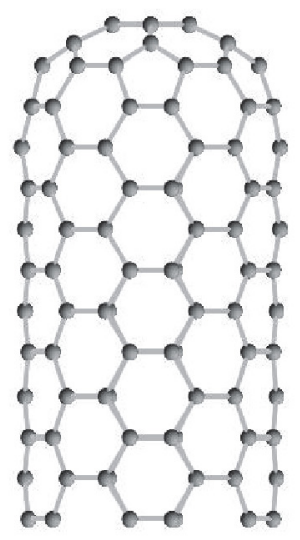

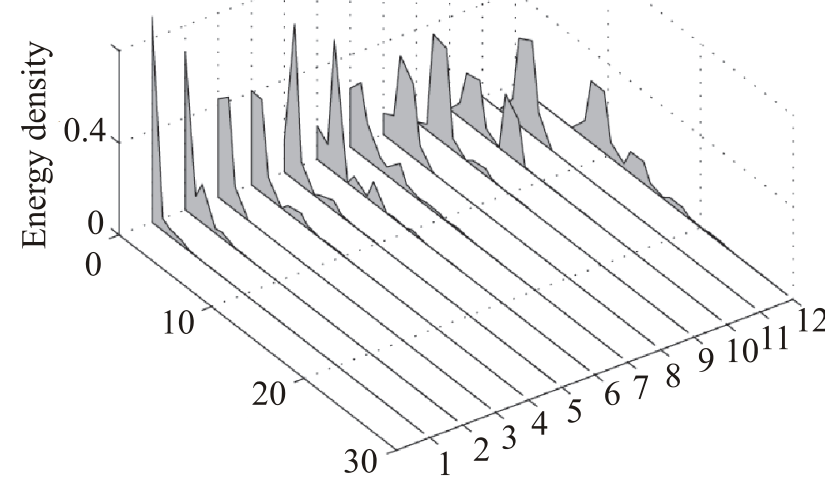

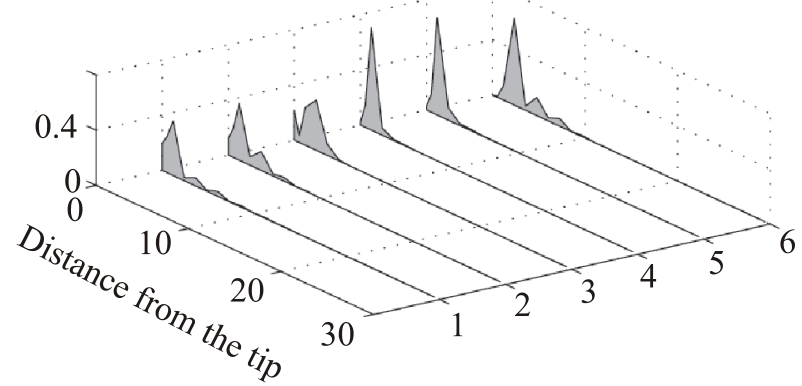

Fig. 3. Localized phononic modes in capped single-wall carbon nanotubes. Left: two examples of the structure of the tips of the singlewalled capped carbon nanotubes with the chirality indices $(6,0)$ and $(6,6)$. Right: Distribution of the energy density $p(x)$ of localized modes along the nanotube for the capped nanotubes $(6,0)$ and $(6,6)$, respectively; $x$ is the distance from the nanotube tip, and $n$ is the mode number. For the nanotube with the chirality $(6,0)$, the localized modes have frequencies of $\omega=1522.0,527.9,1389.6,601.9$, $297.5,1145.7,510.3,1498.1,262.9,499.3,148.0$, and $1487.2 \mathrm{~cm}(n=1,2, \ldots, 12)$. For the nanotube with the chirality $(6,6)$, the frequencies are $\omega=556.7,538.7,1260.5,1591.2,1586.7$, and $548.0 \mathrm{~cm}(n=1, \ldots, 6)[21]$. 
The armchair nanotube $(m, m)$ with the index $m=6$ has $L \geqslant 4$ transverse segments with $N=2 m+2 m L+2 m$ carbon atoms (with $2 m$ atoms in the tip and the segment). The structure with the smallest number of atoms $N=72$ for $m=6$ and $L=4$ is transformed into fullerene $C_{72}$.

In our numerical studies we employ the interaction potentials usually employed for modeling the dynamics of large macromolecules [20-22]. To describe oscillations of the nanotube, the system Hamiltonian can be presented in the form [21]

$$
H=\sum_{n=1}^{N}\left[\frac{1}{2} M\left(\dot{\mathbf{u}}_{n}, \dot{\mathbf{u}}_{n}\right)+P_{n}\right],
$$

where $M$ is the mass of the carbon atom, $\mathbf{u}_{n}=$ $=\left[x_{n}(t), y_{n}(t), z_{n}(t)\right]$ is the radius-vector of the $n$th carbon atom at the time $t$. The last term describes the energy of interaction of $n$th atom with the neighboring atoms,

$$
P_{n}=\frac{1}{2} \sum_{j=1}^{3} V_{j}+\sum_{j=1}^{3} U_{j}+\sum_{j=1}^{3} W_{j} .
$$

The first three terms in Eq. (5) describe the deformation energy due to a direct interaction between pairs of atoms (valent bonds), the next three terms describe the deformation energy of the valent angles, and finally, the last three terms describe the deformation energy associated with a change in the effective angle between the planes created by the valent bonds. The details of the models can be found the earlier paper [22].

The largest number of surface localized modes is found in the nanotube $(6,0)$. If we number those modes in accordance with their degree of localization (see Fig. 3, right), we can divide all the modes into three groups. The first group consists of the modes localized at the very edge of the nanotube (the modes $n=1,2,3,4$ ), which can be related to the oscillations of the hexagon structure of atoms at the tip.

In-plane oscillations of the hexagon generate the most localized modes $(n=1,2)$. Out-of-plane oscillations involve the neighboring atoms, and the corresponding modes are less localized (modes 3 and 4). The second group of modes (the modes $n=5,6,7,8$ ) is associated with the oscillations of the second transverse segment of atoms, and such oscillations are less localized. Finally, the third group of modes corresponds to the localized oscillations shifted away from the edge of the nanotube (the modes $n=9,10$, $11,12)$. These modes practically do not involve oscillations of the tip atoms.

Strongly localized modes observed in the nanotube $(6,6)$ are not related directly to the modes of a hexagon at the tip, so the number of such modes is two times smaller. As seen in Fig. 3, the oscillations are localized at the two edge segments of atoms and do not penetrate deeper into the structure. Therefore, other types of nanotubes and car- bon structures are expected to support strongly localized surface states.

In addition, we studied strongly anharmonic surface modes localized at the tips. Unlike the linear modes discussed above, which are exact solutions of the linearized equations of motion, the anharmonic modes radiate phonons decaying in a finite time. A nonlinear mode with the largest lifetime that we found is the torsion mode of the nanotube $(6,0)$ with frequency of $527.9 \mathrm{~cm}^{-1}$. For the input kinetic energy of $3.04 \mathrm{eV}$ (mode amplitude $A=70 \AA$ ) and temperature $T=0$, the decay time of this mode (calculated when the mode loses half of its energy) was found to exceed $100 \mathrm{ps}$, while it is smaller for a finite temperature, e.g., it is $20 \mathrm{ps}$ for $T=30 \mathrm{~K}$ and 9 ps for $T=300 \mathrm{~K}$. Generally, the tips of capped nanotubes may support all types of long-lived anharmonic modes, which generalize the linear modes but demonstrate a finite lifetime of a few picosecond (at room temperatures)

Thus, in this example we observe that small-amplitude oscillations of single-walled capped carbon nanotubes can support phonon oscillatory surface states localized at the tips of the capped nanotubes, in analogy to the surface states discussed above and known to exist in solids and optics due to defect but here being supported by curved surface of the tip. In the linear regime, such phonon modes appear as exact vibrational eigenmodes of the structures, but they also survive in the anharmonic regime and demonstrate long lifetime due to emission of phonons.

\section{Concluding remarks}

In this paper, we have discussed several examples of very dissimilar physical systems where localized edge modes appear due to different physical mechanisms, such as nonlinearity, topology, or curvature.

First, we have demonstrated that nonlinearity can support different types of localized modes near the edge of a semi-infinite discrete array of nonlinear particles. We have discussed the mechanism of nonlinearity-induced stabilization and existence of such modes above a certain power threshold. Importantly, a similar analysis can be applied to other types of nonlinear discrete surface modes, such as flat-topped modes and twisted modes, as well as staggered modes in defocusing waveguides.

Second, we have discussed how topology can support a novel type of topological edge states even in the linear regime. To achieve this, the straight chain should be transformed into a zigzag array that demonstrates nontrivial topological properties. A general theoretical concept has been verified by the proof-of-principle microwave experiments with dielectric Mie-resonant spherical particles, with the ability to control the subwavelength topologically protected electromagnetic edge modes by changing the polarization of the incident wave. 
Finally, we have discussed an example of localized phonon oscillations in single-walled carbon nanotubes, where curvature can support the energy localization which occurs at capped tips of the nanotubes. Such modes are somewhat similar to the edge states of semi-infinite arrays of optical waveguides, and they appear due to the curvature-induced effective trapping potential.

\section{Acknowledgments}

I acknowledges useful collaborations and discussions with his colleagues and collaborators, especially Mario Molina, Alexander Poddubny, Alexander Savin, and Alexey Slobozhanyuk. This work was supported by the Strategic Fund of the Australian National University.

1. P. Yeh, A. Yariv, and A.Y. Cho, Appl. Phys. Lett. 32, 104 (1978).

2. A.D. Boardman, P. Egan, F. Lederer, U. Langbein, and D. Mihalache, In: Nonlinear Surface Electromagnetic Phenomena, V.M. Agranovich, A.A. Maradudin, H.-E. Ponath, and G.I. Stegeman (eds.), Elsevier Science Publishers, New York, (1991), p. 73.

3. Y.S. Kivshar, Laser Phys. Lett. 5, 703 (2008).

4. M.I. Molina, R.A. Vicencio, and Y.S. Kivshar, Opt. Lett. 31, 1693 (2006).

5. K.G. Makris, S. Suntsov, D.N. Christodoulides, G.I. Stegeman, and A. Hache, Opt. Lett. 30, 2466 (2005).

6. Y.S. Kivshar and G.P. Agrawal, Optical Solitons: From Fibers to Photonic Crystals, Academic Press (2003).

7. S. Suntsov, K.G. Makris, D.N. Christodoulides, G.I. Stegeman, A. Haché, R. Morandotti, H. Yang, G. Salamo, and M. Sorel, Phys. Rev. Lett. 96, 063901 (2006).

8. C.R. Rosberg, D.N. Neshev, W. Krolikowski, A. Mitchell, R.A. Vicencio, M.I. Molina, and Y.S. Kivshar, Phys. Rev. Lett. 97, 083901 (2006).

9. M.Z. Hasan and C.L. Kane, Rev. Mod. Phys. 82, 3045 (2010).

10. L. Lu, J.D. Joannopoulos, and M. Soljaĉiĉ, Nature Photonics 8, 821 (2014).

11. A. Poddubny, A. Miroshnichenko, A. Slobozhanyuk, and Y. Kivshar, ACS Photonics 1, 101 (2014).

12. A. Slobozhanyuk, A.N. Poddubny, A.E. Miroshnichenko, P.A. Belov, and Y.S. Kivshar, Phys. Rev. Lett. 114, 123901 (2015).

13. S. Kruk, A. Slobozhanyuk, D. Denkova, A. Poddubny, I. Kravchenko, A. Miroshnichenko, D. Neshev, and Y. Kivshar, Small 13, 1603190 (2017).

14. S. Kruk, A. Poddubny, D. Smirnova, L. Wang, A. Slobozhanyuk, A. Shorokhov, I. Kravchenko, B. LutherDavies, and Y. Kivshar, Nature Nanotechnology 14, 126 (2019).
15. S.Q. Shen, Topological Insulators. Dirac Equation in Condensed Matters, Springer, Heidelberg (2013).

16. M. Atala, M. Aidelsburger, J.T. Barreiro, D. Abanin, T. Kitagawa, E. Demler, and I. Bloch, Nature Phys. 9, 795 (2013).

17. R.S. Savelev, A.P. Slobozhanyuk, A.E. Miroshnichenko, Y.S. Kivshar, and P.A. Belov, Phys. Rev. B 89, 035435 (2014).

18. S.G. Davidson and M. Steslicka, Theory of Surface States, Oxford Science, New York (1996).

19. Physical Properties of Carbon Nanotubes, R. Saito, G. Dresselhaus, and M.S. Dresselhaus (eds.), Imperial College Press, London (1998).

20. D.W. Noid, B.G. Sumpter, and B. Wunderlich, Macromolecules 24, 4148 (1991).

21. A.V. Savin and Y.S. Kivshar, Appl. Phys. Lett. 94, 111903 (2009).

22. A.V. Savin and Y.S. Kivshar, Europhys. Lett. 82, 66002 (2008).

\section{Локалізовані крайові моди в дискретних фотонних} та фононних системах

(Огляд)

\section{Ю. Ківшар}

В огляді обговорюються приклади трьох різних фізичних систем, локалізація енергії в яких відбувається поблизу країв внаслідок нелінійності, топології або кривизни. У багатьох таких системах існування локалізованих крайових мод та їх властивості можуть бути перевірені експериментально, як, наприклад, в разі масивів слабкозв'язаних оптичних хвилеводів.

Ключові слова: граничні режими, нелінійність, топологія, дискретні системи.

\section{Локализованные краевые моды в дискретных фотонных и фононных системах (Обзор) \\ Ю. Кившарь}

В обзоре обсуждаются примеры трех различных физических систем, локализация энергии в которых происходит вблизи краев вследствие нелинейности, топологии или кривизны. Во многих таких системах существование локализованных краевых мод и их свойства могут быть проверены экспериментально, как, например, в случае массивов слабосвязанных оптических хвильоводів.

Ключевые слова: граничные моды, нелинейность, топология, дискретные системы. 